\title{
A Power Law for the Distortion of Planar Sets
}

\author{
Michael H. Freedman* \\ Department of Mathematics, University of California, San Diego, La Jolla, CA 92093, USA
}

\begin{abstract}
We consider how to map the sites of a square region of planar lattice into a three-dimensional cube, so as to minimize the maximum distortion of distance. We consider the cube to be endowed with a "foliated" geometry in which horizontal distance is standard but vertical communication only occurs at the surface of the cube. These geometries may naturally arise if a planar data set is to be stored in a stack of chips. It is proved that any one-to-one map which fills the cube with a fixed "density" must produce a distortion of distance which grows as the one-sixth power of the diameter of the square and the two-thirds power of the density. Moreover, we explicitly define one-to-one maps with $100 \%$ density, one-sixth power stretching, and a small leading coefficient. As a final note, a high-dimensional analog is considered.
\end{abstract}

Let $S_{x}=\left\{\left(x_{0}, y_{0}\right) \mid x_{0}, y_{0} \in Z\right.$ and $\left.0 \leq x_{0} \leq x-1,0 \leq y_{0} \leq x-1\right\}$ be the square of side $=x$ in the usual square lattice. Similarly, denote the cube by

$$
C_{y}=\left\{\left(x_{0}, y_{0}, z_{0}\right) \mid x_{0}, y_{0}, z_{0} \in Z \text { and } 0 \leq x_{0}, y_{0}, z_{0} \leq y-1\right\}
$$

In $S_{x}$ we take the "street-metric" $\left\|\left(x_{0}, y_{0}\right)\right\|=\left|x_{0}\right|+\left|y_{0}\right|$ and in the cube $C_{y}$ a "foliation-metric." 1 This is defined for $p, q \in C_{y}$ by setting $\operatorname{dist}(p, q)=$ $\min ($ \# (horizontal bonds in $\gamma$ ) $+w \#$ (vertical bonds in $\gamma)$ ). The minimum is taken over all "admissible" paths from $p$ to $q$. We say $\gamma$ is admissible if it is a union of bonds between adjacent lattice sites and contains no vertical segment (parallel to the $z$-axis) except along the exterior of the cube. We have introduced a constant weighting factor $w$ to relate horizontal and vertical distance in assessing the length of $\gamma$. Assume that $y \geq x^{2 / 3}$, then there will exist injections $f: S_{x} \rightarrow C_{y}$.

\footnotetext{
* Supported by DARPA Contract 86-A227500.

${ }^{1}$ See the final paragraph for an observation of J. Komlós regarding the choice of target metric.
} 
The general problem is to determine the largest constant $K(x, y)$ so that any injection $f: S_{x} \rightarrow C_{y}$ will have "stretching" $s_{f} \geq K$, that is, $\operatorname{dist}[f(p), f(q)] \geq$ $K(x, y)$ for some $p, q \in S_{x}$ with $\operatorname{dist}(p, q)=1$. We would also like to describe the injections which realize this minimum stretching, $K(x, y)$. The asymptotic relations between $x, y$, and $K$ are of particular interest. For this reason we introduce the notion of density $\rho=x^{2} / y^{3}$ and establish the following lower bound on the stretching $s$ in terms of $\rho, w$, and $x$.

Theorem. Let $w$ be the vertical weight factor, and let $\rho=x^{2} / y^{3}$ be the density. For any one-to-one map $f: S_{x} \rightarrow C_{y}$, we have the following bound on stretching: $s_{f}(x)=$ $s(x)>(2 \sqrt{6})^{-1} w^{1 / 2} \rho^{2 / 3} x^{1 / 6}$ whenever $x$ is sufficiently large with respect to $w$. In general the bound is $s(x)>(2 \sqrt{6})^{-1} w^{1 / 2}\left(\rho^{4 / 3} x^{1 / 3}-\rho^{2 / 3} x^{-1 / 3}\right)^{1 / 2}$, whenever the righthand side is a real number.

Proof. Let the inverse image of the ith level square of $C_{y}$ be denoted $T_{i}$, $f^{-1}(z=i)=T_{i}, 0 \leq i \leq y-1$. We think of $T_{i}$ as the ith country of $S_{x}$. We define $F_{i} \subset T_{i}$ to be those points distance whose distance is one from a point of some $T_{j}, i \neq j$. We construct the dual graph $D$ by taking a vertex for each nonempty $T_{i}$ and a (single) $(i, j)$-edge between $T_{i}$ and $T_{j}$ iff the distance between the two countries is equal to one. Give $D$ the usual edge-path metric and let $\pi: S_{x} \rightarrow D$ assign each point to its country. We will define several numerical quantities associated with the geometry of the inverse image picture of $f$; the proof consists of formulating four inequalities and one identity among these numbers and solving them to obtain a bound on stretching.

Let $d=\left(1 / x^{4}\right) \sum_{\alpha, \beta \in S_{x}} \operatorname{dist}_{D}(\pi(\alpha), \pi(\beta))$. That is, $d$ is the average over pairs of points in $S_{x}$ of their distance in the dual graph. We have

$$
s d>\frac{1}{3} w\left(\rho^{2 / 3} x^{2 / 3}-1\right) \quad \text { (vertical estimate) }
$$

Adjacent countries in $S_{x}$ must map to levels whose separation is $<s / w$ and, similarly, if $\operatorname{dist}_{D}\left(T_{i}, T_{j}\right)=d_{i j}$, then $|i-j|<(s / w) d_{i j}$. Averaging over pairs of points of $S_{x}$ we see that $s d / w$ must be greater to or equal to the average vertical separation of image points. Because of the discrete setting, it is easy to check that this last quantity is minimized when $f\left(S_{x}\right)$ is a horizontal slab in $C_{y}$ of dimensions $y \times y \times \rho y, y=\rho^{-1 / 3} \times x^{2 / 3}$. In this case the average vertical separation (by a computation of Archimedes) is at least $\frac{1}{3}\left(\rho^{2 / 3} x^{2 / 3}-1\right)$, establishing (1).

Define $p=(1 / y)\left(\sum_{i=0}^{y-1} \operatorname{card}\left(F_{i}\right)\right)$ to be the average number of points in the frontier. We have

$$
p<4 s \rho^{-1 / 3} x^{2 / 3} \quad \text { (horizontal estimate) }
$$

Actually, for each $i, 0 \leq i \leq y-1, \operatorname{card}\left(F_{i}\right)<4 s \rho^{-1 / 3} x^{2 / 3}$, since a square array of side $\rho^{-1 / 3} x^{2 / 3}$ has fewer than $4 s \rho^{-1 / 3} x^{2 / 3}$ lattice points closer than distance $=s$ to the array's boundary, and only these points may be the image of a frontier point without producing at least $s+w$ stretching of some frontier bond. 
Now consider the vertical $\mathbf{V}(x=$ const.) and horizontal $\mathbf{H}$ ( $y=$ const.) families of lines through $S_{x}$. Both $\mathbf{V}$ and $H$ consist of $x$ lines of length $x$. (It is convenient to take the length to be $x$, not $x-1$, by extending each line half a unit on each side of the square. $)$ Define $\#=(1 / y)\left(\sum_{i=0}^{y-1} \#_{i}\right)$ where $\#_{i}$ is the number of lines in $\mathbf{V} \cup \mathbf{H}$ that $T_{i}$ meets, that is, \# is the average number of horizontal and vertical line fragments in each country. Roughly, this number is small if the countries are squarish and large is they are oblong. Define $e_{k}^{v}$ to be the number of $T_{i}$ that the $k$ th vertical line meets and $e_{k}^{\mathrm{H}}$ to be the number of $T_{i}$ the $k$ th vertical line meets, $0 \leq k \leq x-1$. We set $e=(1 / 2 x)\left(\sum_{k=0}^{x-1} e_{k}^{V}+\sum_{k=0}^{x-1} e_{k}^{H}\right)$ to be the number of countries an average line in $\mathbf{V} \cup \mathbf{H}$ meets. We obtain

$$
\# \leq 2 p \quad \text { (perimeter bounds arc number) }
$$

and

$$
2 e>d \quad \text { (average sectional diameter bounds average diameter). }
$$

First, suppose $T_{i}$ does not contain all the lattice points on antire line of $V \cup H$. If $l \in V \cup H$ and $l \cap T_{i} \neq \varnothing$, then the intersection must contain at least one frontier point and that point lies on only two lines (one from $V$ and one from $H)$. Thus $2 \operatorname{card}\left(F_{i}\right)$ dominates the number of line fragments in $T_{i}$. On the other hand, if $T_{i}$ contains an entire line of $V \cup H$, it is easy to see that $\operatorname{card}\left(F_{i}\right) \geq x$, whereas the number of lines of $\mathbf{V} \cup \mathbf{H}$ that can meet $T_{i}$ is, of course, bounded by $2 x$. Averaging over $i$ yields (3).

To justify (4) consider a pair of points $\left(x_{0}, y_{0}\right),\left(x_{1}, y_{1}\right) \in S_{x}$. We apply the triangle inequality to the images in the dual graph $D$ :

$$
\begin{aligned}
\operatorname{dist}_{D}\left(\pi\left(x_{0}, y_{0}\right)\right. & \left., \pi\left(x_{1}, y_{1}\right)\right) \\
\leq & \operatorname{dist}_{D}\left(\pi\left(x_{0}, y_{0}\right), \pi\left(x_{0}, y_{1}\right)\right)+\operatorname{dist}_{D}\left(\pi\left(x_{0}, y_{1}\right), \pi\left(x_{1}, y_{1}\right) .\right.
\end{aligned}
$$

Averaging this inequality over all pairs of points in $S_{x}$ we get

$$
d \leq \underline{e}^{\mathrm{V}}+\underline{e}^{\mathrm{H}},
$$

where $e^{v}\left(e^{\mathrm{H}}\right)$ is the average (over all pairs of points in $S_{x}$ with the same $x$-coordinate $(y$-coordinate)) distance in $D$ between two points which lie on a vertical (horizontal) line. Grouping the distances to be averaged into those along single horizontal or vertical lines we see that these subaverages $e_{k}^{v}$ and $e_{k}^{\mathrm{H}}$ are strictly dominated by the respective $e_{k}^{\mathrm{V}}$ and $e_{k}^{\mathrm{H}}$ 's. Thus $d<(1 / x)\left(\sum_{k=0}^{x-1} e_{k}^{\mathrm{V}}+\sum_{k=0}^{x-1} e_{k}^{\mathrm{H}}\right)=2 e$.

We come to

$$
\# \frac{x}{e}=2 \rho^{1 / 3} x^{4 / 3} \quad \text { (area identity). }
$$

Notice that

$$
\frac{x}{e}=\frac{x}{(1 / 2 x)\left(\sum e_{k}^{\mathrm{V}}+\sum e_{k}^{\mathrm{H}}\right)}=\frac{2 x^{2}}{\sum e_{k}^{\mathrm{V}}+\sum e_{k}^{\mathrm{H}}}=\frac{2 x^{2}}{y \#}
$$


Since $y=\rho^{-1 / 3} x^{2 / 3}$, we compute

$$
\# \frac{x}{e}=\frac{\# 2 x^{2}}{y \#}=\frac{2 x^{2}}{\rho^{-1 / 3} x^{2 / 3}} .
$$

To finish we use (1)-(5) to solve for $s$ as follows: substitute (3) and (4) into (5) to obtain

$$
4 p \frac{x}{d}>2 \rho^{1 / 3} x^{4 / 3}
$$

Substituting (2) for $p$ in the above yields

$$
16 s \rho^{-1 / 3} x^{5 / 3}>2 \rho^{1 / 3} x^{4 / 3} d \text { or } d<8 s \rho^{-2 / 3} x^{1 / 3} \text {. }
$$

Now substitute this estimate for $d$ into (1) to get

$$
s^{2}>\frac{w}{24} \rho^{4 / 3} x^{1 / 3}-\frac{w}{24} \rho^{2 / 3} x^{-1 / 3}
$$

If the second term is sufficiently small with respect to the square root of the first, we obtain the simple formula $s(x)>(1 / 2 \sqrt{6}) w^{1 / 2} \rho^{2 / 3} x^{1 / 6}$. If not, we must settle for the general estimate.

To summarize, the proof merely balances the following tradeoff: if $s$ is small, the dual graph must have large (average) diameter which implies some countries must be long and thin. These countries will have such a large frontier, some frontier points will be mapped far from the cube's vertical surface-forcing the stretching to be large.

We give a construction of an injection $F: S_{x} \rightarrow C_{y}$ in the extreme case $y=x^{3 / 2}$, $x$ and $y$ integers. For convenience, we will assume $x^{1 / 6}$ is also an integer, and never hesitate to divide or extract roots-this simplification does not distort the important points. Let $\sigma, \sigma^{\prime}$, and $\sigma^{\prime \prime}$, when applied to an odd number, act as the involutions which reverse the ordered sets $\left\{0,1, \ldots, x^{2 / 3}-1\right\},\left\{0,1, \ldots, x^{5 / 6}-1\right\}$, and $\left\{0,1, \ldots, x^{1 / 2}-1\right\}$, respectively. Applied to an even number, $\sigma, \sigma^{\prime}$, and $\sigma^{\prime \prime}$ act as the identity.

Let $R_{x, y}=\left\{\left(x_{0}, y_{0}\right) \mid x_{0}, y_{0} \in Z\right.$ and $0 \leq x_{0} \leq x-1$ and $\left.0 \leq y_{0} \leq y-1\right\}$. Define $h: R_{x^{5 / 6}, x^{1 / 2} \rightarrow S_{x^{2 / 3}}}$ by $h(p, q)=\left(\sigma\left(p_{0}\right)\left[p_{1}\right], x^{1 / 6} q+p_{0}\right)$, where $p=p_{0} x^{2 / 3}+p_{1}$, $0 \leq p_{1} \leq x^{2 / 3}-1$. The map $h$ is a bijection which stretches by no more than a factor of $x^{1 / 6}$. Now set

$$
F(u, v)=\left(h\left(\sigma^{\prime}\left(u_{0}\right)\left[u_{1}\right], \sigma^{\prime \prime}\left(v_{0}\right)\left[v_{1}\right]\right), u_{0}+x^{1 / 6} v_{0}\right),
$$

where $u=u_{0} x^{5 / 6}+u_{1}, 0 \leq u_{1} \leq x^{5 / 6}-1$, and $v=v_{0} x^{1 / 2}+v_{1}, 0 \leq v_{1} \leq x^{1 / 2}-1$. It may be verified that a pair of adjacent lattice sights $(p, q ; \operatorname{dist}(p, q)=1)$ in $S_{x}$ are carried to lattice sights whose distance is

$$
\operatorname{dist}(F(p), F(q)) \leq \max \left(x^{1 / 6}, 2\left(x^{1 / 6}-1\right)+w x^{1 / 6}\right) .
$$


It appears that $F$ is reasonably efficient when $w$ is close to 1 . In general, the analogous $F$ should be defined where the level rectangles $R_{x^{3 / 6}, x^{1 / 2}}$ are replaced by

$$
R_{\theta x^{5 / 0}, x^{1 / 2} / \theta}, \quad \text { where } \theta \text { minimizes } 2\left(\theta x^{1 / 6}-1\right)+\frac{w x^{1 / 6}}{\theta}
$$

Differentiating we find $\theta=w^{1 / 2} / \sqrt{2}$.

When $x^{1 / 6}$ is large, the maximum stretching approaches $2 \sqrt{2} w^{1 / 2} x^{1 / 6}$. These modified $F$ 's appear to be optimal.

To understand $F$, one should compare it with the more obvious ways of folding a square into a cube. Consider "double pleating": pleat a square $x \times x \times 1$ into a rectangular solid $x \times x^{2 / 3} \times x^{1 / 3}$. Now a second perpendicular system of pleats gives an $x^{2 / 3} \times x^{2 / 3} \times x^{2 / 3}$ cube. The first set of pleats causes no stretching but-in what amounts to a finite analog of Gauss' Theorema Egregium-the second transverse set of pleats must distort intrinsic geometry, i.e., cause stretching-in this case by $2 w x^{1 / 3}$. Employing shifts and reflections instead of "folds" in the second pleating, stretching $=w x^{1 / 3}$ can be obtained, and the formula for this map is

$$
G(u, v)=\left(\sigma\left(u_{0}\right)\left[u_{1}\right], \sigma\left(v_{0}\right)\left[v_{1}\right], u_{0}+x^{1 / 3} v_{0}\right)
$$

where

$$
\begin{array}{ll}
u=u_{0} x^{2 / 3}+u_{1}, & 0 \leq u_{1} \leq s^{2 / 3}-1, \\
v=v^{0} x^{2 / 3}+v_{1}, & 0 \leq v_{1} \leq x^{2 / 3}-1 .
\end{array}
$$

Imagine the case $C_{y}$ as foliated by squares at height $z=$ constant. The inverse images $G^{-1}$ (horizontal squares) are themselves squares. Think of these as forming a "country-map" of $S_{x}$ with dual graph $D$. Recall the definiton of $d$ from the proof of the theorem. The estimate (1) applied to $G$ gives $s_{3}^{2}\left(x^{1 / 3}-1\right) \geq w\left(x^{2 / 3}-1\right)$, or essentially $s>3 w x^{1 / 3} / 2$. This is unnecessarily large. The idea in constructing $F$ is to share stretching between the first and second pleatings so as to make $d$ larger-on the order of $x^{1 / 2}$. The cost is that the level sets are not square but rectangular. These considerations have been balanced in the definition of $F$. There is essentially $x^{1 / 6}$ stretching both between adjacent countries and within each country. It is the sum (with weights) of these two stretchings that controls the maximum stretching of $F$.

The reader should compare the following description of $F$ with the preceding formula: stretch $S_{x}$ by a factor of $x^{1 / 6}$ along the $y$-axis. Then "pleat" (with "pleat" lines parallel to the $y$-axis) into $x^{1 / 3}$ sheets (regard the sheets in groups of size $x^{1 / 6}$ as filling a single horizontal level so that an $x^{7 / 6} \times x^{2 / 3} \times x^{1 / 6}$ rectangular solid results). Second stage "pleating" yields an $x^{2 / 3} \times x^{2 / 3} \times x^{2 / 3}$ cube.

The construction of $F$ has an amusing - but probably unimportant-generalization to maps $F^{n}: S_{x} \rightarrow H_{x^{2 / n}}^{n}$ from the square of side $x$ to the $n$-dimensional hypercube of side $x^{2 / n}$. The injections $F^{n}$ appear to minimize (up to multiplicative 


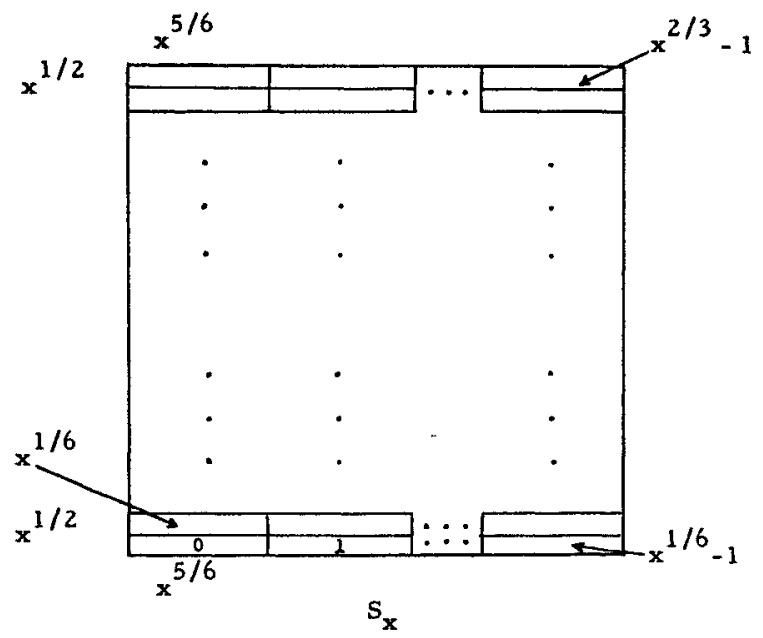

Fig. 1. The level sets of $F$.

constants) stretching between our standard planar metric and the metric on $H_{x^{2 / n}}^{n^{2}}$ which records the length of edge paths which are allowed to move parallel to the $k$ th coordinate axis only if some smaller coordinate $j<k$ is extremal, $n \geq k>2$.

One proceeds recursively to pack rectangles into hypercubes, each subcube

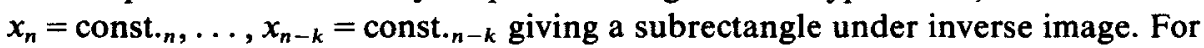
the procedure (yet to be defined), let $s(r, t ; k)$ be the stretching that occurs when the rectangle $R_{r, s}$ is packed into a $k$-cube. In packing $R_{a_{n} x, x / a_{n}}$ into an $n$-cube, a balance between internal (within subrectangles) and external (between subrectangles) stretching is obtained when

$$
\frac{x^{1 / n}}{a_{n} a_{n-1}}=s\left(a_{n-1} x^{n-1 / n}, \frac{x^{n-1 / n}}{a_{n-1}} ; n-1\right) .
$$

This happens when the subrectangles are

$$
\boldsymbol{R}_{a_{n-1} x^{(n-1) / n}, x^{(n-1) / n} / a_{n-1}}
$$

At the next level of subcube, balance is obtained when

$$
\frac{x^{1 / n}}{a_{n} a_{n-1} a_{n-2}}=s\left(a_{n-2} x^{(n-1) / n}, \frac{x^{(n-2) / n}}{a_{n-2}} ; n-2\right) .
$$

Continuing in this way we obtain a sequence of conditions which terminates with

$$
\frac{x^{1 / n}}{a_{n} a_{n-1} \cdots a_{2}}=s\left(a_{2} x^{2 / n}, \frac{x^{2 / n}}{a_{2}} ; 2\right)=a_{2} .
$$




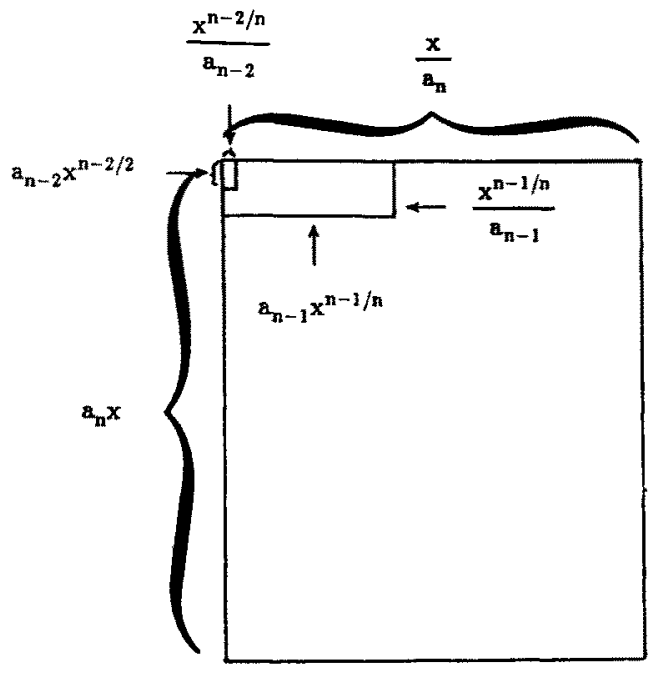

Fig. 2

Furthermore, if $a_{n}$ is regarded as constant and $a_{n-1}$ variable, the solution of the first equation is $a_{n-1}=s\left(a_{n} x, x / a_{n} ; n\right)$. In this fashion, one may recursively obtain formulae for $s\left(a_{n} x, x / a_{n} ; n\right)$ which when specialized to $a_{n}=1$ (the case of mapping from a square $s_{x}$ ) give the following results for the exponential rate of growth of $s$ as a function of $x$ :

\begin{tabular}{lcccccc}
\hline & $n=2$ & $n=3$ & $n=4$ & $n=5$ & $n=6$ & $n=7$ \\
\hline Stretching for $F^{n}$ as a power of $x$ & 0 & $1 / 6$ & 0 & $2 / 15$ & $1 / 15$ & $13 / 105$ \\
\hline
\end{tabular}

(We have not included the explicit formula for $F^{n}, n>3$.)

We remark briefly that: (1) sources of dimension $>2$ have not been treated here, but the same methods would apply, (2) the exact lattices used (e.g., square) only enter superficially into our discussion, and (3) there is no analogous phenomenon when the target (hyper-) cube is given a more isotropic metric. In this case, János Komlós has shown me injections of $S_{x}$ for which the stretching does not increase with $x$ (even when density $=1$ ).

\section{Acknowledgments}

I would like to thank Benedict Freedman and János Komlós for interesting discussions on the subject of this note.

Received September 29, 1986. 